Research article

Open Access

\title{
p66 Shc and tyrosine-phosphorylated Shc in primary breast tumors identify patients likely to relapse despite tamoxifen therapy
}

\author{
A Raymond Frackelton Jr'1,2, Li Lu ${ }^{3}$, Pamela A Davol ${ }^{1}$, Robert Bagdasaryan ${ }^{4}$, Laurie J Hafer ${ }^{5}$ and \\ Dennis C Sgroi6
}

\author{
1Department of Research, Roger Williams Medical Center, 825 Chalkstone Avenue, Providence, RI 02908, USA \\ 2Departments of Medicine and of Pathology and Laboratory Medicine, Brown University, Box G-RWH, Providence, RI 02912, USA \\ ${ }^{3}$ Department of Surgery, Roger Williams Medical Center, 825 Chalkstone Avenue, Providence, RI 02908, USA \\ ${ }^{4}$ Department of Pathology, Roger Williams Medical Center, 825 Chalkstone Avenue, Providence, RI 02908, USA \\ ${ }^{5}$ Catalyst Oncology, Inc., One Innovation Drive, Worcester, MA 01605, USA \\ ${ }^{6}$ Molecular Pathology Unit and Center for Cancer Research, Massachusetts General Hospital, Building 149, 13th Street, Charlestown, MA 02129, \\ USA;Mailstop: Molecular Pathology Unit - 7149
}

Corresponding author: A Raymond Frackelton, A_Frackelton_Jr@brown.edu

Received: 1 Sep 2006 Revisions requested: 2 Oct 2006 Revisions received: 17 Nov 2006 Accepted: 29 Dec 2006 Published: 29 Dec 2006

Breast Cancer Research 2006, 8:R73 (doi:10.1186/bcr1631)

This article is online at: http://breast-cancer-research.com/content/8/6/R73

(c) 2006 Frackelton et al.; licensee BioMed Central Ltd.

This is an open access article distributed under the terms of the Creative Commons Attribution License (http://creativecommons.org/licenses/by/2.0), which permits unrestricted use, distribution, and reproduction in any medium, provided the original work is properly cited.

\begin{abstract}
Introduction Shc adapter proteins are secondary messenger proteins involved in various cellular pathways, including those mediating receptor tyrosine kinase signaling and apoptosis in response to stress. We have previously reported that high levels of tyrosine-phosphorylated Shc (PY-Shc) and low levels of its inhibitory p66 Shc isoform are strongly prognostic for identifying both early node-negative and more advanced, node-positive, primary breast cancers with high risk for recurrence. Because aberrant activation of tyrosine kinases upstream of Shc signaling proteins has been implicated in resistance to tamoxifen - the most widely prescribed drug for treatment of estrogen receptorpositive breast cancer - we hypothesized that Shc isoforms may identify patients at increased risk of relapsing despite tamoxifen treatment.
\end{abstract}

Methods Immunohistochemical analyses of PY-Shc and p66 Shc were performed on archival primary breast cancer tumors from a population-based cohort (60 patients, 9 relapses) and, for validation, an independent external cohort (31 patients, 13 relapses) in which all patients received tamoxifen as a sole systemic adjuvant prior to relapse.

Results By univariate and multivariate analyses, the Shc proteins were very strong and independent predictors of treatment failure in both the population-based cohort (interquartile hazard ratio $=8.3,95 \%$ confidence interval $[\mathrm{Cl}]$ 1.8 to $38, P=0.007$ ) and the validating cohort (interquartile relative risk $=12.1,95 \% \mathrm{Cl} 1.7$ to $86, P=0.013$ ).

Conclusion These results suggest that the levels of PY-Shc and p66 Shc proteins in primary tumors identify patients at high risk for relapsing despite treatment with tamoxifen and therefore with further validation may be useful in guiding clinicians to select alternative adjuvant treatment strategies.

\section{Introduction}

Tamoxifen, a partial estrogen receptor (ER) antagonist and the most widely prescribed drug for the treatment of ER-positive breast cancer, provides a $40 \%$ to $50 \%$ decrease in the rate of relapse in this group of patients [1]. The precise mechanism of tamoxifen action is unknown; however, binding of tamoxifen to the ER inhibits estradiol activation of the receptor and ERdependent proliferation and promotes cellular apoptosis $[2,3]$. Despite the objective responses or disease stabilization achieved with tamoxifen treatment in more than $50 \%$ of patients with previously untreated metastatic breast cancer [4], nearly $100 \%$ of metastatic patients and up to $40 \%$ of patients receiving adjuvant tamoxifen will relapse and die [1]. Clearly, there is a need to identify those patients who are at

$\Delta \chi^{2}=$ change in chi-squared; $\mathrm{Cl}=$ confidence interval; $\mathrm{df}=$ degrees of freedom; $\mathrm{ER}=$ estrogen receptor; HR $=$ hazard ratio; IHC $=$ immunohistochemistry; LBA = ligand-binding assay; $\mathrm{PLR}=$ partial likelihood ratio; $\mathrm{PR}=$ progesterone receptor; $\mathrm{PY}=$ tyrosine-phosphorylated; $\mathrm{RFS}=$ relapse-free survival; $\mathrm{RR}=$ relative risk. 
increased risk of relapse even though tamoxifen therapy has been administered: such patients would be candidates for chemotherapy or alternative adjuvant therapies such as Her$\operatorname{ceptin}^{\circledR}$ (Genentech, Inc., South San Francisco, CA, USA) and tyrosine kinase inhibitors.

Accumulating evidence indicates that active tyrosine kinases play a major role in innate and acquired tamoxifen resistance $[3,5]$. Ligand-bound ER has the ability to activate growth factor receptors and their downstream kinases through either direct cellular membrane interactions, as occur with HER2 and insulin-like growth factor receptor, or via downstream phosphorylation and transactivation events, as occur with the epidermal growth factor receptor $[6,7]$. Conversely, crosstalk with these receptors enhances the sensitivity of the ER to ligand and may lead to ligand-independent activation [8] or may hypersensitize ER to the normally weak estrogenic effects of tamoxifen [9].

Three Shc adapter proteins (p46, p52, and p66 Shc) are tyrosine-phosphorylated (PY) in response to signaling from ER as well as from these other growth factor receptors [10-12]. The PY-Shc proteins play key roles in activating the Grb2-SOS to Ras to mitogen-activated protein kinase pathway [13-15] and therefore the levels of PY-Shc might correspond to the likelihood of tamoxifen resistance. In contrast to the other Shc isoforms, however, p66 Shc functions as both a feedback downregulator of growth factor signaling $[16,17]$ and as a critical facilitator of stress-induced apoptosis [18]. Thus, decreased tumor expression of p66 Shc may correspond to both increased likelihood of growth factor-mediated tamoxifen resistance and resistance to tamoxifen-driven apoptosis.

We hypothesized, therefore, that high levels of PY-Shc and low levels of p66 Shc in primary tumors may identify patients with increased risk of relapse despite having received tamoxifen therapy. Here, we report an affirmative test of this hypothesis in a population-based cohort and an independent, external validation cohort of patients, all of whom received firstline tamoxifen therapy as a sole systemic adjuvant.

\section{Materials and methods Patient populations}

Population-based cohort

Formalin-fixed, primary tumors from 60 patients (9 relapses) with invasive breast cancer diagnosed between 1985 and 1991 were obtained from the archives of the Department of Pathology, Roger Williams Medical Center (Providence, RI, USA). Patients had surgical removal of the primary lesion (followed by local radiation if breast-conserving surgery was employed), and all patients received tamoxifen as the sole systemic adjuvant therapy until time of censorship or disease recurrence. Of the 60 patients, none was known to be ER-negative and 55 were ER-positive. ER positivity was defined as a dextran-coated charcoal ligand-binding assay (LBA) result of more than $10 \mathrm{fmol} / \mathrm{mg}$ protein [19]. Non-relapsing patients had a mean follow-up of 6.1 years, and relapsing patients had a mean time to first relapse of 3.4 years ( 0.08 to 7.4 years).

\section{Validation cohort}

Archival, formalin-fixed, paraffin-embedded primary tumors from 31 patients (13 relapses) were obtained as a subset of the case-control study by $\mathrm{Ma}$ et al. [20]. All patients received tamoxifen as part of their first course of therapy. No patients received neoadjuvant chemotherapy or other systemic adjuvant therapies before disease recurrence. All patients were ER-positive, defined as more than $10 \mathrm{fmol} / \mathrm{mg}$ protein by dextran-coated charcoal LBA or as an immunohistochemical result of more than $10 \%$ nuclear staining with a score of more than 3 using a monoclonal ER antibody, clone 1D5 (Dako North America, Inc., Carpinteria, CA, USA). To minimize confounding of potential predictive markers by clinicopathological characteristics, relapsing and non-relapsing patients were closely matched by ER/progesterone receptor (PR) status, nodal status, tumor grade, patient age at diagnosis, and tumor size. Non-relapsing patients had a mean follow-up of 8.7 years, and relapsing patients had a mean time to first recurrence of 5.3 years ( 0.5 to 11.2 years).

\section{Antibodies and immunohistochemical analysis}

Characterization of the affinity-purified antibodies to PY-Shc and p66 Shc and the immunohistochemical procedures have been described previously [21]. An average staining intensity for all invasive cancer cells in a section was determined $[22,23]$ : staining intensities of cancer cells were scored on a scale of 0 to 5 [21] by a scorer blinded to patient outcomes and clinicopathological characteristics. To accurately reflect the heterogeneity in the tumor staining, the intensity score was multiplied by the percentage of invasive cancer cells staining at each intensity. These results were summed and then divided by 100 to achieve a scale of 0 (no invasive cancer cells stained) to 5 (all invasive cancer cells stained maximally). From the population-based study, a random subset of slides ( $n=$ 24) was scored independently by a second scorer blinded both to patient outcomes and to the other scorer's results. A concordance of 0.96 between the scorers, with regard to the assignment of patients to high versus low ratios of PY-Shc to p66 Shc staining, was observed, with a Pearson correlation coefficient of $0.80(P<0.0001)$ [21].

\section{Statistical considerations}

Statistical calculations were performed using the Stata statistical package (Stata version 8.0; StataCorp LP, College Station, TX, USA). Clinicopathological characteristics were analyzed by univariate log-rank analysis of their Kaplan-Meier survivor functions. Differences between PY-Shc-to-p66 Shc ratios in relapsing and non-relapsing patients were tested for significance by Wilcoxon rank sum test. The ability of PY-Shc and p66 Shc, as continuous variables, to predict disease recurrence was analyzed in univariate and multivariate Cox 
proportional hazards models with relapse-free survival (RFS) as an endpoint. RFS was defined as the time from surgical removal of the tumor until first clinical recurrence or loss to follow-up (censored). Cox models were checked for validity of proportional hazards assumptions and goodness of fit using Schoenfeld and Cox-Snell residual analyses, respectively. Covariate improvement to models was tested for significance by partial likelihood ratio ( $\mathrm{pLR}$ ) analysis. For visual evaluation of RFS, Kaplan-Meier survival functions were constructed using PY-Shc and p66 Shc values dichotomized on their median values, and differences between the dichotomized
Shc variables were tested by univariate log-rank analysis. All statistical tests were two-sided, with significance taken as $P<$ 0.05 .

\section{Results}

In the population-based cohort, univariate log-rank analysis of RFS identified significant predictive values for nodal status and tumor stage and values approaching significance for tumor grade and PR status. In the validation cohort, however, no clinicopathological characteristic attained predictive significance (Table 1). This was expected inasmuch as the valida-

Table 1

Clinicopathological characteristics and univariate analysis of ability to predict failure of tamoxifen therapy

\begin{tabular}{|c|c|c|c|c|c|c|c|c|}
\hline \multirow[b]{3}{*}{ Variable } & \multicolumn{4}{|c|}{ Population-based cohort } & \multicolumn{4}{|c|}{ Case-control cohort } \\
\hline & \multicolumn{6}{|c|}{ RFS } & \multicolumn{2}{|c|}{ RFS } \\
\hline & $n^{\mathrm{a}}$ & Percentage $^{b}$ & 8 years & $P^{c}$ & $n^{\mathrm{a}}$ & Percentage ${ }^{b}$ & 8 years & $P^{c}$ \\
\hline All patients & $9 / 60$ & 15 & 79 & & $13 / 31$ & 42 & 67 & \\
\hline Nodal status & $9 / 60$ & 15 & & 0.04 & $13 / 28$ & 46 & & 0.30 \\
\hline Negative & $4 / 45$ & 9 & 85 & & $8 / 15$ & 53 & 57 & \\
\hline Positive & $5 / 15$ & 33 & 64 & & $5 / 13$ & 38 & 71 & \\
\hline AJCC stage & $9 / 60$ & 15 & & 0.16 & $\mathrm{ND}^{d}$ & $\mathrm{ND}^{\mathrm{d}}$ & & \\
\hline 1 & $2 / 28$ & 7 & 92 & & & & & \\
\hline $2 a$ & $0 / 3$ & 0 & $100^{e}$ & & & & & \\
\hline $2 b$ & $7 / 27$ & 26 & 58 & & & & & \\
\hline За & $0 / 1$ & 0 & $100^{e}$ & & & & & \\
\hline Tumor stage & $9 / 60$ & 15 & & 0.01 & $13 / 31$ & 42 & & 0.17 \\
\hline 1 & $2 / 32$ & 6 & 93 & & $3 / 12$ & 25 & 74 & \\
\hline 2 & $4 / 21$ & 21 & 55 & & $10 / 19$ & 53 & 62 & \\
\hline 3 & $3 / 4$ & 75 & $50^{e}$ & & & & & \\
\hline 4 & $0 / 1$ & 0 & 100 & & & & & \\
\hline Tumor grade & $9 / 60$ & 15 & & 0.06 & $13 / 31$ & 42 & & 0.56 \\
\hline 1 & $0 / 5$ & 0 & 100 & & $0 / 1$ & 0 & 100 & \\
\hline 2 & $2 / 22$ & 9 & 82 & & $8 / 19$ & 45 & 63 & \\
\hline 3 & $7 / 26$ & 27 & 69 & & $5 / 11$ & 40 & 70 & \\
\hline PR status & $9 / 60$ & 15 & & 0.06 & $13 / 31$ & 47 & & 0.96 \\
\hline Negative & $0 / 16$ & 0 & 100 & & $3 / 6$ & 50 & 67 & \\
\hline Positive & $6 / 36$ & 17 & 71 & & $10 / 25$ & 40 & 67 & \\
\hline Age & $9 / 60$ & 15 & & 0.15 & $13 / 31$ & 42 & & 0.56 \\
\hline$<50$ years & $2 / 6$ & 33 & $83^{e}$ & & $2 / 2$ & 100 & 100 & \\
\hline$\geq 50$ years & $7 / 54$ & 13 & 84 & & $11 / 29$ & 38 & 64 & \\
\hline
\end{tabular}

a9/60 indicates that there were 9 treatment failures out of 60 patients with known values for the indicated clinical characteristic. bThe percentage of treatment failures for the indicated clinical characteristic. ${ }^{c} P$ is given for log-rank (or log-rank trend) univariate analysis. Sensitivity tests conducted with missing values provided no evidence for selection bias. ${ }^{\mathrm{d} C l i n i c a l}$ characteristic was not determined. eFor patients with AJCC stage $2 a$ and $3 a$ disease and patients less than 50 years old, the RFS is reported at 7 years. For patients with tumor stage 3 disease, the RFS is reported at 6 years. AJCC, American Joint Committee on Cancer; PR, progesterone receptor; RFS, relapse-free survival. 
tion cohort was a subset of patients from a case-control study in which relapsing and non-relapsing patients had been closely matched by ER/PR status, nodal status, and tumor size [20]. The case-control validation cohort, therefore, provided a means to evaluate the Shc predictive markers with minimal potential confounding by clinicopathological variables.

Results representative of the immunohistochemical analyses of primary tumors shown in Figure 1a depict the contrast typically observed between PY-Shc and p66 Shc staining intensities in cancer cells from patients who relapsed despite therapy compared to those who did not. Based on a previously established scoring system for Shc staining intensity [21], patients in both studies who relapsed (irrespective of tamoxifen therapy) had primary tumors with a higher PY-Shc-to-p66 Shc staining ratio compared to those tumors from patients who did not relapse $(0.31 \pm 0.10$ versus $0.09 \pm 0.02, P=0.002$, and $0.23 \pm 0.08$ versus $0.05 \pm 0.01, P=0.049$, in the populationbased and validation cohorts, respectively; Figure $1 \mathrm{~b}, \mathrm{c})$.

Consistent with these observations, by univariate Cox proportional hazards analysis, significant increases in risk of relapse in these tamoxifen-treated patients were observed with increasing values of the PY-Shc-to-p66 Shc ratio in each cohort. The increase in risk with PY-Shc-to-p66 Shc ratio can be appreciated by the large hazard ratio (HR) for the full observed range of patient scores: 14 (95\% confidence interval [Cl]) 2.1 to $93, P=0.006$ ) for the population-based cohort and $43(95 \% \mathrm{Cl} 4$ to $480, P=0.002)$ for the validation cohort. Considering the PY-Shc and p66 Shc variables as mutually adjusted covariates, rather than constrained as the ratio, improves model fit considerably [21]. Compared to the ratio in the present study, PY-Shc and p66 Shc considered together as covariates improved model fit (by pLR analysis for the significance of the change in chi-squared $\left[\Delta \chi^{2}\right]$ for $n$ degrees of freedom [ $\Delta \mathrm{df}]$ : pLR $\Delta \chi^{2}=6.08, \Delta \mathrm{df}=1, P=0.013$ ) and provided an HR for the full observed range of Shc values of 240 (95\% Cl 9.8 to $5,900, P=0.001$ ) and an interquartile HR of 8.6 in the population-based cohort (Table 2). For the validation cohort, a full-range HR of $53(95 \% \mathrm{Cl} 1.8$ to $1,600, P=0.02)$ and an interquartile HR of 7.7 were observed (Table 2). This relationship between the Shc scores and relapse in tamoxifentreated patients in both studies is easily visualized from the Kaplan-Meier RFS functions. The median values of the composite PY-Shc and p66 Shc scores from each cohort were used as a cut point to divide the patients into high- and lowrisk groups. Within 8 years of primary diagnosis, 38\% and $50 \%$ of high-risk tamoxifen-treated patients in the populationbased and validation cohorts, respectively, relapsed, whereas only $3.3 \%$ and $14 \%$ of the low-risk patients, respectively, relapsed (Figure 2).
Figure 1

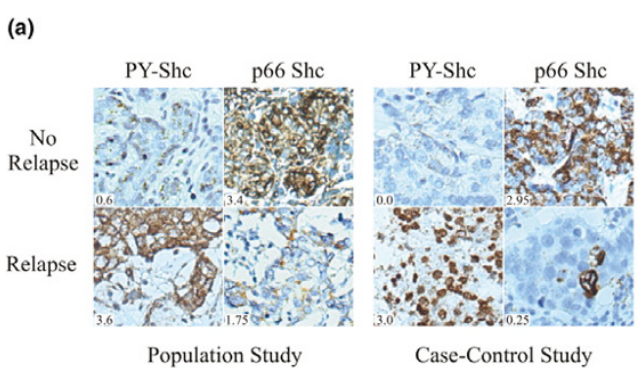

(b)

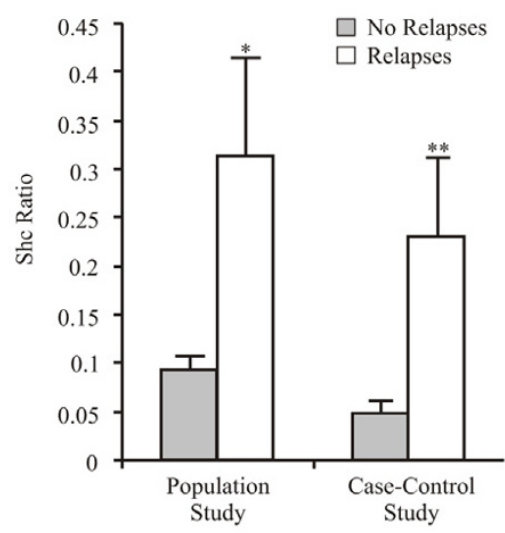

(c)

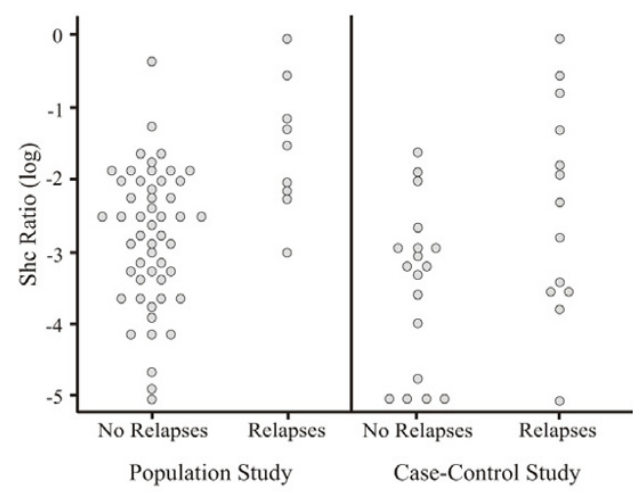

Comparison of the PY-Shc-to-p66 Shc ratio in primary tumors as a function of disease recurrence. (a) Photomicrographs of PY-Shc and p66 Shc immunohistochemical staining patterns in primary tumors from patients who received tamoxifen and then either relapsed or did not relapse. Shc scores are shown in the insets. The left panel shows the staining patterns of two patients from the population-based cohort, and the right panel shows the staining patterns of two patients from the case-control cohort. Total magnification, $\times 200$. (b) The ratio of PY-Shc to p66 Shc (Shc ratio), scaled 0 to 1 , is shown (mean \pm standard error) for primary tumors from patients who either relapsed or did not relapse. A comparison of the mean Shc ratios for patients in the populationbased study with or without relapses is shown on the left side of the column graph (50 no relapses, 9 relapses; $\left.{ }^{\star} P=0.002\right)$. A comparison of the mean Shc ratios for patients in the case-control study with or without relapses is shown on the right side of the column graph (18 no relapses, 13 relapses; ${ }^{\star} P=0.049$ ). (c) Scatter histograms of the Shc ratio, on a log scale, observed in the patients' primary tumors as a function of relapse in the population-based and case-control cohorts. PYShc, tyrosine-phosphorylated Shc. 
Multivariate analyses of ability of Shc proteins to predict failure of tamoxifen therapy

\begin{tabular}{lccc}
\hline & \multicolumn{1}{c}{$\begin{array}{c}\text { Population cohort } \\
\text { Model }\end{array}$} & $\mathrm{HR}^{\mathrm{a}}(95 \% \mathrm{Cl})$ & $\begin{array}{c}\text { Case-control cohort } \\
\mathrm{RR}^{\mathrm{a}}(95 \% \mathrm{Cl})\end{array}$ \\
\hline $\begin{array}{l}\text { PY-Shc, p66 Shc } \\
\text { PY-Shc, p66 Shc } \\
\quad \text { Adjusted for nodal status }\end{array}$ & $8.6(2.6-30)$ & 0.001 & $7.7(1.4-44)$ \\
\hline
\end{tabular}

aHR (interquartile) is the RR of relapse comparing patients in the 75th percentile of PY-Shc and 25th percentile of p66 Shc to patients in the 25th percentile of PY-Shc and 75th percentile of p66 Shc. The multivariate Cox model containing PY-Shc and p66 Shc was adjusted for nodal status, AJCC (American Joint Committee on Cancer) stage (population-based cohort), tumor stage, tumor grade, progesterone receptor status, and patient age at diagnosis. bSignificance using Wald statistic. Cl, confidence interval; HR, hazard ratio; PY-Shc, tyrosine-phosphorylated Shc; RR, relative risk.

Figure 2

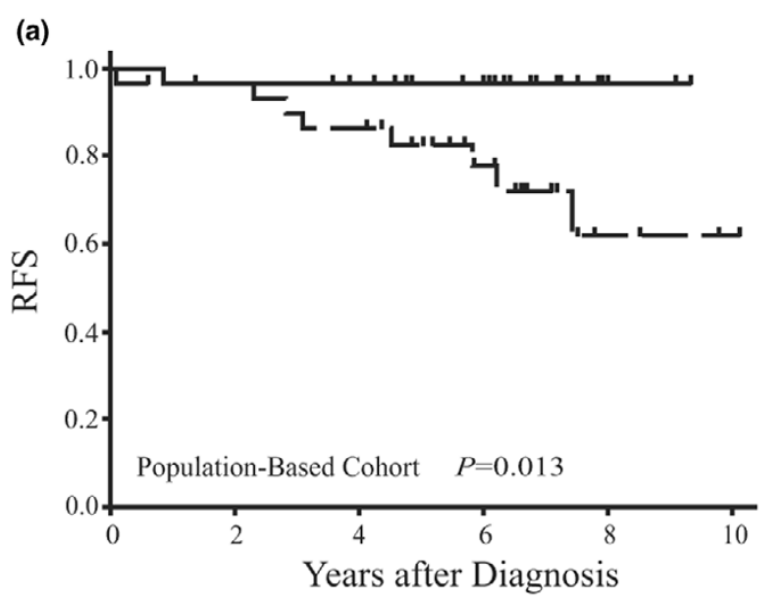

(b)

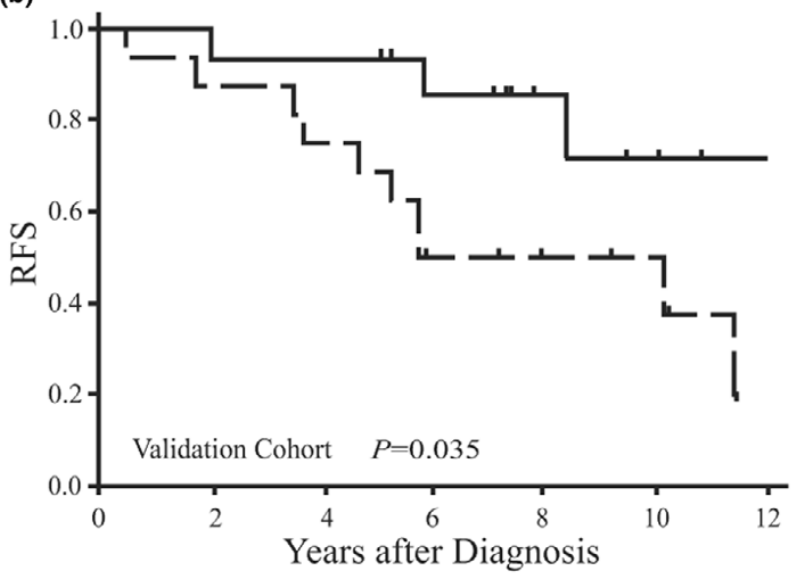

Relapse-free survival (RFS) according to Shc in patients with breast cancer. (a) Kaplan-Meier graphical analysis of RFS in patients from the population-based cohort partitioned by the median value for Shc $(P=$ 0.013 ). (b) Kaplan-Meier graphical analysis of RFS in patients from the case-control cohort partitioned by the median value for Shc $(P=$ $0.035)$.
By multivariate analysis, the ability of the Shc proteins to predict relapse despite tamoxifen therapy was independent of nodal status, AJCC (American Joint Committee on Cancer) stage (population-based cohort), tumor stage, tumor grade, PR status, and patient age at diagnosis. Of these clinical variables, only nodal status remained as a significant covariate (population-based cohort: $P=0.04$; validation cohort: $P=$ 0.04), with the Shc proteins retaining their full prognostic value and strength (Table 2). Inclusion of nodal status improved the overall fit of the model, suggesting that it may be useful as an independent predictive covariate along with PYShc and p66 Shc.

\section{Discussion}

In women with ER-positive breast tumors, the high initial response rate to tamoxifen has made this drug the most widely prescribed treatment for this group of patients. Aberrant activation of kinase signaling pathways in invasive breast cancer cells, however, may play a widespread role in failure of tamoxifen therapy $[3,5]$. Such pathways have the potential to bypass ER-dependent growth of cells or recruit ERs independent of ligand activation through crosstalk, thus facilitating a resistant phenotype while confounding the predictive utility of standard clinical markers. Therefore, it is not surprising that many investigators have focused on identifying new molecular markers that may identify patients with high risk of relapse despite tamoxifen therapy. Supporting such an approach, the application of a multimarker predictive model evaluating the interdependency between ER, PR, HER2/neu, bcl-2, myc, and tp53 has resulted in superior predictive power for failure of tamoxifen therapy, compared to standard marker evaluations alone [22].

In another approach, the expression levels of large numbers of genes have been used to predict disease outcome [23]. Despite the promising results obtained from these microarray gene analyses-based studies, increased costs and complex methodology may limit their widespread application. Additionally, gene-expression analysis does not measure the actual level of protein expression, post-translational modification, or 
aberrant subcellular localization in the invasive cancer cells, potentially limiting this methodology's accuracy for predicting individual patient risk.

In this study, we analyzed the expression and post-translational modification of Shc adapter proteins in two independent cohorts of patients with breast cancer by a semi-quantitative immunohistochemistry (IHC) staining method and found the Shc proteins to be strongly predictive for disease recurrence in tamoxifen-treated patients. Primary tumors of these patients who subsequently relapsed contained characteristically higher levels of PY-Shc, lower levels of p66 Shc protein, or both compared to primary tumors of those patients who did not relapse. In Cox survival models, the levels of PY-Shc and p66 Shc could be modeled as the ratio of PY-Shc to p66 Shc or (more effectively) as linked continuous covariates, showing a very strong ability to predict patient outcome (Table 2). These observations support the hypothesized molecular model of tamoxifen resistance, in which PY-Shc and p66 Shc subserve at least partially opposing molecular functions.

Although the predictive ability of markers is most robust when their expression can be considered as continuous variables [24], categorizing the Shc scores into groups with high and low risk of relapse despite tamoxifen therapy is useful for comparing RFS curves (Figure 2). By univariate log-rank analysis, RFS for high- and low-risk groups in each cohort was significantly different. Tamoxifen-treated patients with tumors having a high PY-Shc and/or low p66 Shc score experienced a significantly shorter RFS compared to tamoxifen-treated patients with tumors having a low PY-Shc and/or high p66 Shc score.

Discussions of multimarker prediction models often present HRs or relative risks (RRs) that represent the full range of risk over which the model can assign patients. A clinically more informative measure, however, is the interquartile RR: the range of risk that can be observed within the middle $50 \%$ of patients. The Shc proteins assigned a very wide range of risk of relapse over the middle $50 \%$ of patients, with an interquartile RR of 8 (Table 2) in the population-based cohort and 12 (Table 2) in the validation cohort. The validation cohort was a subset of the case-controlled patients studied by $\mathrm{Ma}$ et al. [20], and its Shc interquartile RR of 12 is comparable to the $\mathrm{RR}$ of 7 reported by $\mathrm{Ma}$ et al. [20] for the expression ratio of HOXB13/IL-17BR receptor in a multivariate model for these same patients.

In multivariate analysis, the ability of the Shc proteins to predict relapse in tamoxifen-treated patients was strong and independent of all potential covariates. Of these, only nodal status remained as a marginally significant covariate in each cohort, with the Shc proteins retaining their full prognostic value and strength (Table 2). Inclusion of nodal status improved the overall fit of the model, suggesting that it may be useful as a predictive covariate along with PY-Shc and p66 Shc. Consistent with our previous report [21] of the Shc proteins predicting outcome for both node-negative and node-positive patients, neither by analysis of correlation nor interaction in the Cox model was there evidence of association between the Shc proteins and nodal status. The independence of nodal status as a predictor is also consistent with the findings of a large meta-analysis in which node-positive compared to node-negative patients were shown to have approximately twice the absolute risk of failing tamoxifen therapy [1].

Other potential covariates may attain significance when larger study populations are examined. For example, to the extent that HER2/neu overexpression and activation [3,25] correlate with both tamoxifen resistance and its activation signals through Shc-associated pathways, the predictive value of Shc may occur, in part, due to its capacity as a downstream reporter of HER2/neu activity. However, HER2/neu is activated in only approximately $16 \%$ of ER-positive patients [26]. For the majority of cases of tamoxifen resistance, the specific etiology remains unknown, although there is some evidence that tamoxifen-induced apoptosis may play a role [27]. Additionally, in other studies, we have observed that the ability of Shc proteins to predict patient outcome is independent of HER2/neu expression levels (unpublished data).

All of the patients' tumors in these studies expressed ER as determined by LBA. Tumors that are ER-positive by LBA constitute approximately $90 \%$ of breast tumors that are ER-positive, as judged by currently used, more sensitive IHC assays $[28,29]$. It will be important in subsequent large validation studies to determine whether the Shc proteins are similarly able to predict relapse after tamoxifen therapy for this small subgroup of patients whose tumors express low amounts of ER.

The difference in incidence of relapse in the population-based study $(15 \%, 9 / 40)$ and the higher incidence in the validation study $(42 \%, 13 / 31)$ reflects the fact that the validation cohort is a subset of the Ma et al. [20] case-control study, in which equal numbers of relapsing and non-relapsing patients were selected based on closely paired clinicopathological characteristics. This approach was implemented to minimize variable heterogeneity that could potentially confound the analysis of the predictive abilities of Shc proteins. The case-control design provided ample statistical power with a minimal total number of patients in which to validate our primary hypothesis generated from the population-based study: namely, that the Shc proteins identify patients at high risk of relapse despite tamoxifen therapy. However, this selection procedure does not make possible an exact comparison of the magnitude of the predictive abilities of the Shc proteins ( $H R$ and $R R$ ) between the two studies. Despite this, the significant and very strong $\mathrm{RR}$ in the case-control cohort affirms the results of the population-based study showing the predictive independence of 
the Shc proteins from potentially confounding clinicopathological characteristics.

\section{Conclusion}

The very strong and independent ability of the Shc proteins to predict disease relapse despite tamoxifen treatment reported here, coupled with their well-validated ability to predict relapse in untreated patients, provides a compelling argument for validating these results in larger patient cohorts and randomized trials capable of determining the ability of the Shc proteins to predict tamoxifen benefit. Furthermore, compared to gene array-based methodologies, the application of state-specific antibodies in a standardized immunohistochemical analysis provides a practical and cost-effective approach with the advantage of evaluating post-translational protein modifications in addition to expression levels. Upon further successful validation of these results, high PY-Shc and low p66 Shc expression levels in primary tumors should be clinically useful in predicting patients at high risk of relapse despite tamoxifen therapy and thus in identifying those patients who may benefit from adjuvant chemotherapy or targeted therapies such as Herceptin ${ }^{\circledR}$ and tyrosine kinase inhibitors. Conversely, low PYShc and high p66 Shc expression levels in primary tumors may identify patients at low risk of relapse after tamoxifen therapy and thus spare them the costs and toxicities of additional treatments.

\section{Competing interests}

ARF is an employee of Catalyst Oncology, Inc. (Worcester, MA, USA) and a co-inventor on a patent of the IHC assay for Shc. PAD is a co-inventor on a patent of the IHC assay for Shc. LJH is an employee of Catalyst Oncology, Inc. The remaining authors declare that they have no competing interests.

\section{Authors' contributions}

ARF conceived the study, participated in its design and execution and in the drafting of the manuscript, and conducted the statistical analyses. LL, PAD, and LJH participated in IHC staining and analysis and in the drafting of the manuscript. RB had an integral role in the analysis of the IHC staining. DCS participated in the study design and in the acquisition and production of the case-control specimens. All authors read and approved the final manuscript.

\section{Acknowledgements}

Support for this project included Susan G. Komen Breast Cancer Foundation grants BCTR0403184 (ARF) and BCTR0402932 (DCS), U.S. Department of Defense grants BC980415 (ARF) and W81XWH-04-10606 (DCS), National Cancer Institute grant RO1-1CA112021-01 (DCS), an Avon Foundation grant (DCS), and a Department of Research, Roger Williams Medical Center grant (ARF).

\section{References}

1. Early Breast Cancer Trialists' Collaborative Group (EBCTCG): Effects of chemotherapy and hormonal therapy for early breast cancer on recurrence and 15-year survival: an overview of the randomised trials. Lancet 2005, 365:1687-1717.

2. Hayes DF: Tamoxifen: Dr. Jekyll and Mr. Hyde? J Natl Cancer Inst 2004, 96:895-897.

3. Ring A, Dowsett $M$ : Mechanisms of tamoxifen resistance. Endocr Relat Cancer 2004, 11:643-658.

4. Jaiyesimi IA, Buzdar AU, Decker DA, Hortobagyi GN: Use of tamoxifen for breast cancer: twenty-eight years later. J Clin Oncol 1995, 13:513-529.

5. Shou J, Massarweh S, Osborne CK, Wakeling AE, Ali S, Weiss H, Schiff R: Mechanisms of tamoxifen resistance: increased estrogen receptor-HER2/neu cross-talk in ER/HER2-positive breast cancer. J Natl Cancer Inst 2004, 96:926-935.

6. Kelly MJ, Levin ER: Rapid actions of plasma membrane estrogen receptors. Trends Endocrinol Metab 2001, 12:152-156.

7. Razandi M, Alton G, Pedram A, Ghonshani S, Webb P, Levin ER: Identification of a structural determinant necessary for the localization and function of estrogen receptor alpha at the plasma membrane. Mol Cell Biol 2003, 23:1633-1646.

8. Bunone G, Briand PA, Miksicek RJ, Picard D: Activation of the unliganded estrogen receptor by EGF involves the MAP kinase pathway and direct phosphorylation. Embo J 1996, 15:2174-2183.

9. Osborne CK, Shou J, Massarweh S, Schiff R: Crosstalk between estrogen receptor and growth factor receptor pathways as a cause for endocrine therapy resistance in breast cancer. Clin Cancer Res 2005, 11:865s-870s.

10. Ravichandran KS: Signaling via Shc family adapter proteins. Oncogene 2001, 20:6322-6330.

11. Filardo EJ, Thomas P: GPR30: a seven-transmembrane-spanning estrogen receptor that triggers EGF release. Trends Endocrinol Metab 2005, 16:362-367.

12. Zhang Z, Kumar R, Santen RJ, Song RX: The role of adapter protein Shc in estrogen non-genomic action. Steroids 2004, 69:523-529.

13. Rozakis-Adcock M, McGlade J, Mbamalu G, Pelicci G, Daly R, Li W, Batzer A, Thomas S, Brugge J, Pelicci PG, et al.: Association of the Shc and Grb2/Sem5 SH2-containing proteins is implicated in activation of the Ras pathway by tyrosine kinases. Nature 1992, 360:689-692.

14. Pelicci G, Lanfrancone L, Grignani F, McGlade J, Cavallo F, Forni G, Nicoletti I, Grignani F, Pawson T, Pelicci PG: A novel transforming protein (SHC) with an $\mathrm{SH} 2$ domain is implicated in mitogenic signal transduction. Cell 1992, 70:93-104.

15. Segatto O, Pelicci G, Giuli S, Digiesi G, Di Fiore PP, McGlade J, Pawson T, Pelicci PG: Shc products are substrates of erbB-2 kinase. Oncogene 1993, 8:2105-2112.

16. Migliaccio E, Mele S, Salcini AE, Pelicci G, Lai KM, Superti-Furga G, Pawson T, Di Fiore PP, Lanfrancone L, Pelicci PG: Opposite effects of the p52shc/p46shc and p66shc splicing isoforms on the EGF receptor-MAP kinase-fos signalling pathway. Embo $J$ 1997, 16:706-716.

17. Okada S, Kao AW, Ceresa BP, Blaikie P, Margolis B, Pessin JE: The $66-\mathrm{kDa}$ Shc isoform is a negative regulator of the epidermal growth factor-stimulated mitogen-activated protein kinase pathway. J Biol Chem 1997, 272:28042-28049.

18. Migliaccio E, Giorgio M, Pelicci PG: Apoptosis and aging: role of p66(Shc) redox protein. Antioxid Redox Signal 2006, 8:600-608.

19. Pertschuk LP, Eisenberg KB, Carter AC, Feldman JG: Heterogeneity of estrogen binding sites in breast cancer: morphologic demonstration and relationship to endocrine response. Breast Cancer Res Treat 1985, 5:137-147.

20. Ma XJ, Wang Z, Ryan PD, Isakoff SJ, Barmettler A, Fuller A, Muir B, Mohapatra G, Salunga R, Tuggle JT, et al:: A two-gene expression ratio predicts clinical outcome in breast cancer patients treated with tamoxifen. Cancer Cell 2004, 5:607-616.

21. Davol PA, Bagdasaryan R, Elfenbein GJ, Maizel AL, Frackelton AR $\mathrm{Jr}$ : Shc proteins are strong, independent prognostic markers for both node-negative and node-positive primary breast cancer. Cancer Res 2003, 63:6772-6783.

22. Linke SP, Bremer TM, Herold CD, Sauter G, Diamond C: A multimarker model to predict outcome in tamoxifen-treated breast cancer patients. Clin Cancer Res 2006, 12:1175-1183.

23. Brenton JD, Carey LA, Ahmed AA, Caldas C: Molecular classification and molecular forecasting of breast cancer: ready for clinical application? J Clin Oncol 2005, 23:7350-7360. 
24. Bossard N, Descotes F, Bremond AG, Bobin $Y$, De Saint Hilaire $P$, Golfier F, Awada A, Mathevet PM, Berrerd L, Barbier Y, Esteve J: Keeping data continuous when analyzing the prognostic impact of a tumor marker: an example with cathepsin $D$ in breast cancer. Breast Cancer Res Treat 2003, 82:47-59.

25. DiGiovanna MP, Stern DF, Edgerton SM, Whalen SG, Moore D 2nd, Thor AD: Relationship of epidermal growth factor receptor expression to ErbB-2 signaling activity and prognosis in breast cancer patients. J Clin Oncol 2005, 23:1152-1160.

26. Arpino G, Weiss H, Lee AV, Schiff R, De Placido S, Osborne CK, Elledge RM: Estrogen receptor-positive, progesterone receptor-negative breast cancer: association with growth factor receptor expression and tamoxifen resistance. J Natl Cancer Inst 2005, 97:1254-1261.

27. Mandlekar S, Kong AN: Mechanisms of tamoxifen-induced apoptosis. Apoptosis 2001, 6:469-477.

28. Magne N, Toillon RA, Castadot P, Ramaioli A, Namer M: Different clinical impact of estradiol receptor determination according to the analytical method: a study on 1940 breast cancer patients over a period of 16 consecutive years. Breast Cancer Res Treat 2006, 95:179-184.

29. Pichon MF, Broet $P$, Magdelenat $H$, Delarue JC, Spyratos F, Basuyau JP, Saez S, Rallet A, Courriere P, Millon R, Asselain B: Prognostic value of steroid receptors after long-term follow-up of 2257 operable breast cancers. $B r /$ Cancer 1996, 73:1545-1551. 